\title{
NUEVAS POBLACIONES DE BERBERIDOPSIS CORALLINA HOOK.F. (BERBERIDOPSIDALES: BERBERIDOPSIDACEAE) EN LA REGION DEL BIO BIO, CHILE: UBICACION Y CONSERVACION DE SU HABITAT
}

\section{NEW POPULATIONS FOR BERBERIDOPSIS CORALLINA HOOK.F. (BERBERIDOPSIDALES: BERBERIDOPSIDACEAE) IN BIO BIO REGION, CHILE: LOCATION AND HABITAT CONSERVATION}

\author{
Diego Alarcón' ${ }^{1}$ Cecilia Smith-Ramírez², Paulina Hechenleitner \\ Pablo Ramírez de Arellano ${ }^{4}$, Mario Oliva ${ }^{5}$, Misael Pinto ${ }^{5}$ \\ ${ }^{1}$ Programa de Bosque Nativo, Bioforest S.A., Camino a Coronel, km 15 s/n. Concepción, Chile. \\ diego.alarcon@arauco.cl \\ ${ }^{2}$ Fundación Senda Darwin, Casilla 114-D, Santiago, Chile. \\ ${ }^{3}$ Instituto de Silvicultura, Universidad Austral de Chile, Casilla 567, Valdivia, Chile. \\ ${ }^{4}$ College of Environmental Science and Forestry, State University of New York, Syracuse, 401 Illick Hall, Syracuse, \\ New York, 13210, U.S.A. \\ ${ }^{5}$ Unidad de Mensura Forestal, Bosques Arauco S.A., Los Horcones s/n, Arauco, Chile.
}

\begin{abstract}
RESUMEN
Se presenta la ubicación de 123 nuevos registros de la enredadera leñosa michay rojo o voqui pilfuco (Berberidopsis corallina Hook.f.) en la Región del Bío Bío, Chile, y se describe el estado de conservación de su hábitat. Las poblaciones de esta especie amenazada fueron descubiertas en 65 fragmentos de vegetación y bosques nativos con fines de protección, al interior de predios de la empresa forestal Bosques Arauco S.A. Estos registros incrementan significativamente la distribución espacial y altitudinal conocida para esta especie endémica del país y considerada en peligro de extinción. Además, estos nuevos registros revelan una mayor continuidad de sus poblaciones en la región respecto de datos previos.
\end{abstract}

Palabras claves: Michay rojo, Berberidopsis corallina, especies amenazadas, distribución natural, Cordillera de Nahuelbuta.

\section{ABSTRACT}

The location of 123 new records of the woody climbing vine michay rojo or voqui pilfuco (Berberidopsis corallina Hook.f.) in Bio Bio Region, Chile, is presented and its habitat conservation status is described. The populations of this threatened species were discovered in 65 native forest fragments for conservation purposes in Bosques Arauco S.A. company lands. These records increase significantly the known spatial and altitudinal distribution for this endemic species considered as endangered. They also show a greater proximity among its populations in Bio Bio Region than previous data.

KEYwords: Michay rojo, Berberidopsis corallina, endangered species, natural distribution, Nahuelbuta. 


\section{INTRODUCCION}

A contar de 2002, la empresa forestal Bosques Arauco S.A. y la empresa de investigación Bioforest S.A. iniciaron un proyecto de prospección de la biodiversidad contenida en los bosques nativos existentes de sus predios con plantaciones de Pinus radiata D.Don y diversas especies de Eucalyptus sp. Este desarrollo de información ha tenido un énfasis en la validación y localización espacial de diversas especies de flora nativa amenazada, dentro de las cuales Berberidopsis corallina Hook.f. (Berberidopsidaceae) se considera una de las que presenta mayor grado de amenaza. Esta información generada es utilizada por la empresa en la planificación y gestión productiva de sus plantaciones en el marco de su sistema de gestión ambiental, para asegurar la conservación de las poblaciones de las especies amenazadas.

El uso histórico de la tierra en la costa de la Región del Bío Bío ha determinado que en la actualidad exista un paisaje caracterizado por un gradiente de fragmentos de bosques nativos de gran tamaño y mayor diversidad estructural hacia las zonas de mayor altitud de la Cordillera de Nahuelbuta, hasta una mezcla de terrenos de uso productivo silvoagropecuario con fragmentos más pequeños de bosques o vegetación nativa secundaria, hacia altitudes más bajas en la vertiente oceánica. Es precisamente en este gradiente donde se encuentran los puntos anteriormente descritos de B. corallina en la región.

Conocida como michay rojo, voqui pilfuco o voqui fuco, $B$. corallina es una trepadora leñosa endémica de la cordillera de la Costa de Chile central, con una distribución amplia pero discontinua, que abarca desde la Provincia de Cauquenes en la Región del Maule, latitud $35^{\circ} 58^{\prime}$ sur, hasta la Provincia de Osorno en la Región de Los Lagos, latitud $40^{\circ} 50^{\prime}$ sur (Smith-Ramírez et al. 2005).

B. corallina fue clasificada en la categoría En Peligro de Extinción en el simposio de flora nativa arbórea y arbustiva amenazada de Chile (Serra $e t$ al. 1986, Benoit 1989), al igual que en la lista roja de plantas amenazadas de la Unión Internacional de la Conservación de la Naturaleza UICN (Walter \& Gillett 1998). Por su parte, Hechenleitner et al.
(2005) emplearon la metodología de UICN versión 3.1 (2001), clasificando a B. corallina como En Peligro. Igualmente la Comisión Nacional del Medio Ambiente CONAMA en su proceso de aplicación del Decreto Supremo 75 de 2005 del Ministerio Secretaría General de la Presidencia de la República de Chile, ha propuesto para esta especie la categoría En Peligro (CONAMA 2005). Los argumentos para dichas clasificaciones incluirían observación o estimación que el tamaño de sus poblaciones se habrían reducido en un $80 \%$ o más, durante los últimos diez años o tres generaciones, debido a una disminución en su área de ocupación, o extensión de su presencia o calidad de su hábitat (Hechenleitner et al.2005), estimándose que el área de ocupación de la especie es menor a $10 \mathrm{~km}^{2}$, se halla severamente fragmentado y sus poblaciones continuarían disminuyendo en extensión, área de ocupación, número de localidades o subpoblaciones y número de individuos maduros (Hechenleitner $e t$ al. 2005, CONAMA 2005). Según las clasificaciones mencionadas, la especie se estaría enfrentando a un riesgo alto de extinción en estado silvestre.

Berberidopsis corallina ha sido clasificada en la categoría mencionada, de acuerdo a la información disponible hasta entonces, por presentar una distribución muy discontinua y restringida a sectores costeros, con escasas poblaciones registradas. De acuerdo al número de individuos observados, las principales poblaciones se ubican en la Provincia de Arauco y San Juan de la Costa en la Provincia de Osorno, el área que ocupa esta última población ha sido estimada en 2.000 ha (Smith-Ramírez et al. 2005). Otras poblaciones incluyen aquellas ubicadas en la Reserva Nacional Los Queules en la Región del Maule con sólo dos individuos y Villa Las Araucarias en la Región de la Araucanía.

Dada su discutida posición taxonómica y evolutiva dentro de las plantas con flores, la especie presenta un alto interés científico (APG II 2003, Ronse De Craene 2004).

B. corallina es usada principalmente con fines ornamentales (Riedemann \& Aldunate 2004), y en el sector de San Juan de la Costa, sus vástagos constituyen un recurso importante para la confección de cestería tradicional huilliche (SmithRamírez 1995). 
El objetivo del presente estudio ha sido describir la presencia de $B$. corallina en las poblaciones descubiertas, mediante el proyecto mencionado de prospección de biodiversidad en términos de ubicación, superficie registrada con la especie y conservación del hábitat en cada fragmento de vegetación o bosque nativo que contienen las poblaciones halladas de esta trepadora amenazada.

\section{MATERIALES Y METODOS}

\section{Sitio de ESTUdio}

Esta investigación se desarrolló en los predios de plantaciones forestales de Bosques Arauco S.A., que incluyen vegetación de protección y bosques nativos en una proporción cercana al $24 \%$ de su superficie total. El área donde se detectó la presencia de $B$. corallina corresponde a la vertiente occidental de la cordillera de Nahuelbuta. Esta zona se encuentra cubierta principalmente por plantaciones de $P$. radiata, con presencia de bosques nativos de protección ubicados en sitios de pendientes medias a altas y a lo largo de cursos de ríos y esteros. Los suelos son de profundidad delgada a media. Las precipitaciones son principalmente invernales con 1.300 a $1.900 \mathrm{~mm}$ anuales, variación dada por el gradiente norte-sur. La temperatura media anual oscila alrededor de los $13^{\circ} \mathrm{C}$ (Hajek \& di Castri 1975).

\section{REgisTRo DE LA VEGETACIÓN NATIVA}

La vegetación y bosques nativos en la zona de estudio han sido identificados en fotografías aéreas de escala 1:10.000, en concordancia con la cartografía de la empresa. Los fragmentos del territorio, correspondientes a bosque o vegetación nativa, fueron delimitados y clasificados en las fotografías aéreas en distintas unidades homogéneas de vegetación, de acuerdo a su estructura forestal, principales especies arbóreas dominantes y diferencias topográficas observables en las imágenes. Luego de esta delimitación, se visitaron aquellas unidades representativas de la variabilidad observada en las fotografías.

Estos puntos representativos fueron caracterizados en terreno a través de transectos de $50 \mathrm{~m}$ de longitud, en los cuales se registró estructura forestal, cobertura de copas de los árboles dominantes según las clases: denso (75 a $100 \%$ ), semidenso (50 a $75 \%$ ) o abierto (25 a 50\%), altura total del dosel dominante (según las clases: menor a $2 \mathrm{~m}$, de 2 a $4 \mathrm{~m}$, de 4 a $8 \mathrm{~m}$, de 8 a $12 \mathrm{~m}$, de 12 a 20 m, de 20 a 32 m, y mayor a 32 m) y la lista de especies de flora leñosa registrada en cada transecto. Así, el tipo de información resultante al emplear este método es concordante con el entregado por el catastro nacional de bosque nativo (CONAF-CONAMA-BIRF 1997), pero con un mayor nivel de detalle geográfico. Además, en cada transecto, se registró el grado de cobertura vegetal correspondiente a cada especie de flora leñosa, según el rango de altura de dosel en la cual tiene presencia. Mediante estos registros se detectó la presencia de la enredadera B. corallina, registrándose el rango de cobertura vegetal de esta especie para cada porción de bosque nativo caracterizado.

A partir de la información registrada, las unidades fueron clasificadas en comunidades o tipos forestales definidos por CONAF-CONAMA-BIRF 1997. Cada unidad de bosque nativo fue además referenciada geográficamente en la cartografía digital de la empresa, a partir de la cual se determinó su altitud en base a un modelo de elevación digital que utiliza la empresa.

\section{NIVEL DE CONSERVACIÓN DEL HÁBITAT}

Cada fragmento de bosque con $B$. corallina, fue clasificado según la información registrada de estructura de la comunidad forestal y la cobertura de copas del rodal. Los fragmentos constituidos por bosques adultos o adulto-renovales densos o semidensos, se consideraron de un alto grado de conservación del hábitat, por integrar bosques con nula o muy baja intervención antrópica aparente por extracción maderera. Los bosques adultos o adulto-renovales abiertos y renovales densos y semidensos, se consideraron como de un nivel medio de conservación del hábitat, debido a que su estructura incompleta permite suponer alteraciones de probable origen antrópico. Los renovales abiertos y matorrales fueron clasificados de bajo grado de conservación del hábitat, ya que conforman estructuras presumiblemente asociadas a disturbios recientes o sujetas a presiones de uso en un pasado reciente. Esta clasificación preliminar puede servir como base para determinar prioridades de conservación y necesidad de restauración de los hábitat de $B$. corallina. 


\section{RESULTADOS}

Se registró y localizó la presencia de $B$. corallina en 123 puntos contenidos en 65 fragmentos de bosques o vegetación nativa en propiedades de Bosques Arauco S.A. Los registros se concentran geográficamente en la vertiente costera de la Cordillera de Nahuelbuta, aproximadamente entre la localidad de Lota y al sur del lago Lanalhue (Fig. 1).

Al observar la ubicación detallada de los puntos de registro de la especie (Tabla I), se aprecia que en la zona de estudio, las poblaciones de $B$. corallina se desarrollan a una altitud entre 100 y 885 ms.n.m., encontrándose un $77 \%$ de los registros entre los 200 y 600 ms.n.m., rango en el cual se halla un 94\% de la superficie registrada con la especie. El rango altitudinal entre 300 y 400 ms.n.m. constituye la mayor concentración de vegetación con B. corallina, con un 53\% de la superficie (Fig. 2).
Las poblaciones de michay rojo encontradas integran una amplia gama de tipos de bosque (Tabla I). La mayoría de los puntos se presenta en comunidades del tipo forestal siempreverde, subtipo siempreverde (30\% de los registros), y diversos subtipos del tipo forestal roble-raulícoihue: un $20 \%$ en subtipo roble-raulí-coihue, $20 \%$ en subtipo coihue y $15 \%$ en subtipo roble (Fig. 3). Por último y con menor frecuencia $(7 \%)$, se presenta en los renovales de canelo del tipo forestal siempreverde, mientras que también existe en una situación excepcional dentro del tipo forestal esclerófilo (1\%), de características transicionales hacia comunidades forestales de roble-raulí-coihue. Otros registros, en tanto, se presentaron en situaciones de matorral con otras especies arbustivas no correspondientes a las categorías de tipo forestal según CONAF-CONAMA-BIRF 1997 (Fig. 3).

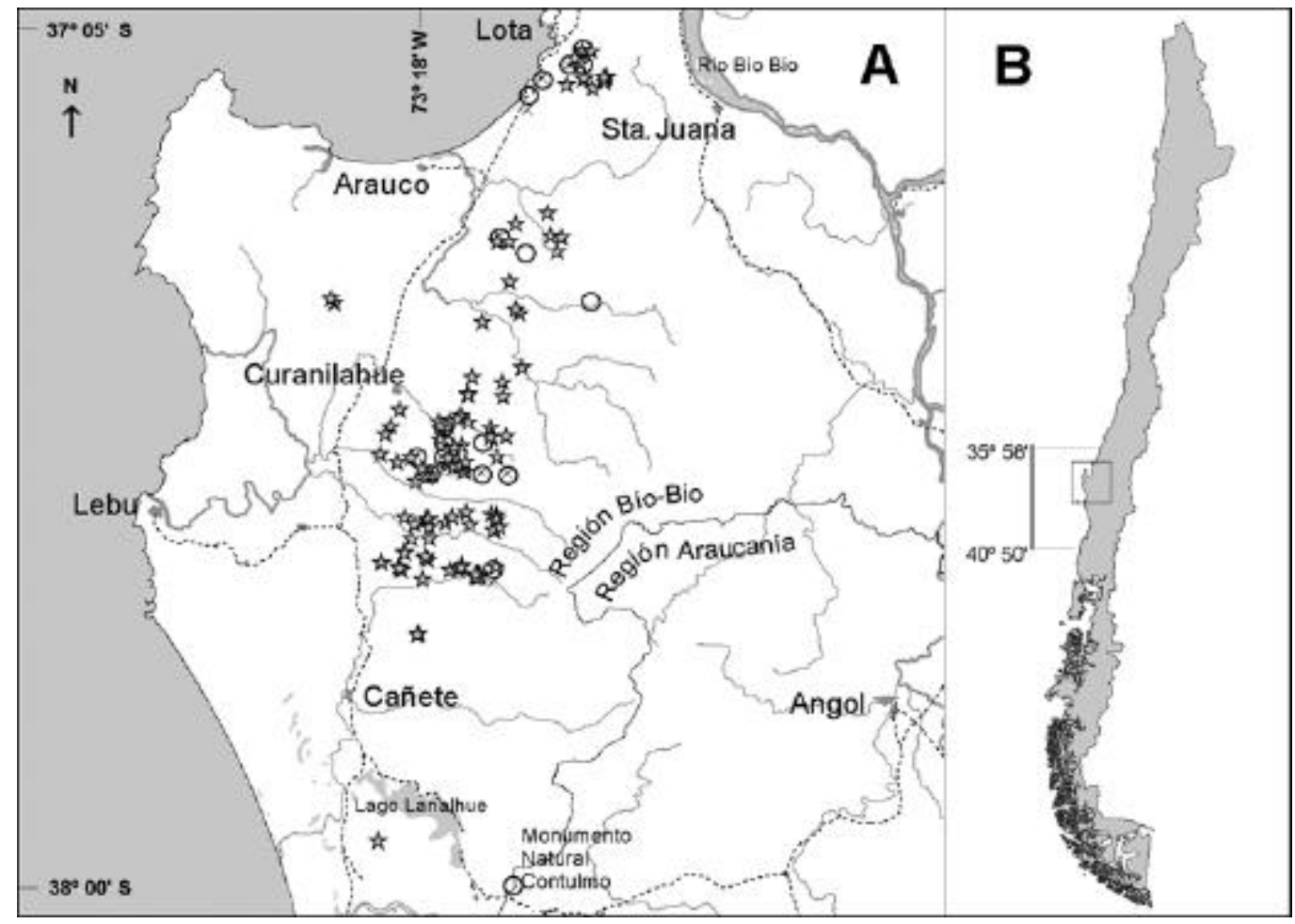

FIgURA 1. a) Ubicación de nuevos registros de B. corallina (estrellas) y distribución en relación a puntos anteriormente conocidos para la especie según Smith-Ramírez et al. 2005 (círculos) y Le Quesne et al. 2000 (cruces). b) Rango latitudinal de distribución natural de B. corallina y zona de estudio en la Región del Bío Bío, Chile.

FIgURE 1. a) New localities for B. corallina (stars) and previously known records for the species according to SmithRamírez et al. 2005 (circles) and Le Quesne et al. 2000 (crosses). b) Latitudinal position of B. corallina natural distribution and study area in Bio Bio Region, Chile. 
Berberidopsis corallina: nuevas poblaciones: ALARCón, D. ETAL

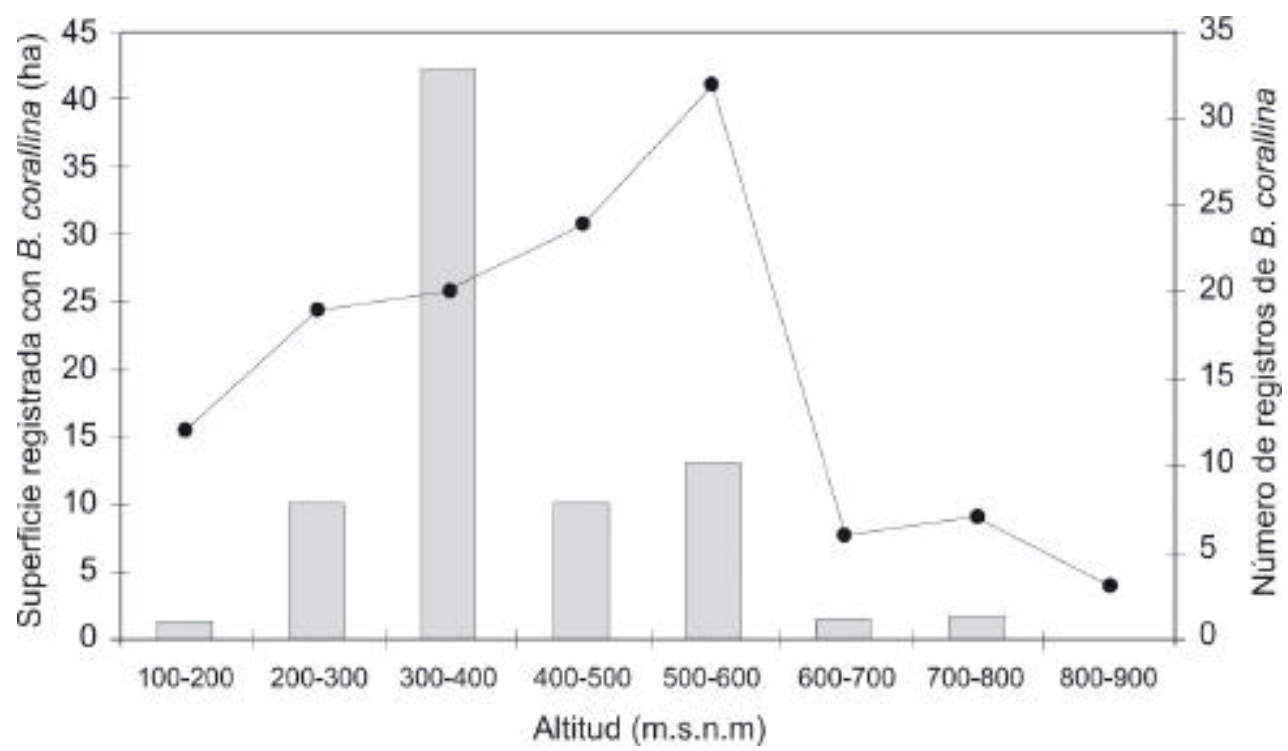

Figura 2. Distribución de los registros de B. corallina según altitud, tanto en superficie (barras) como en número de registros (línea).

FiguRE 2. Number of records (line) and area (strips) for B. corallina records.

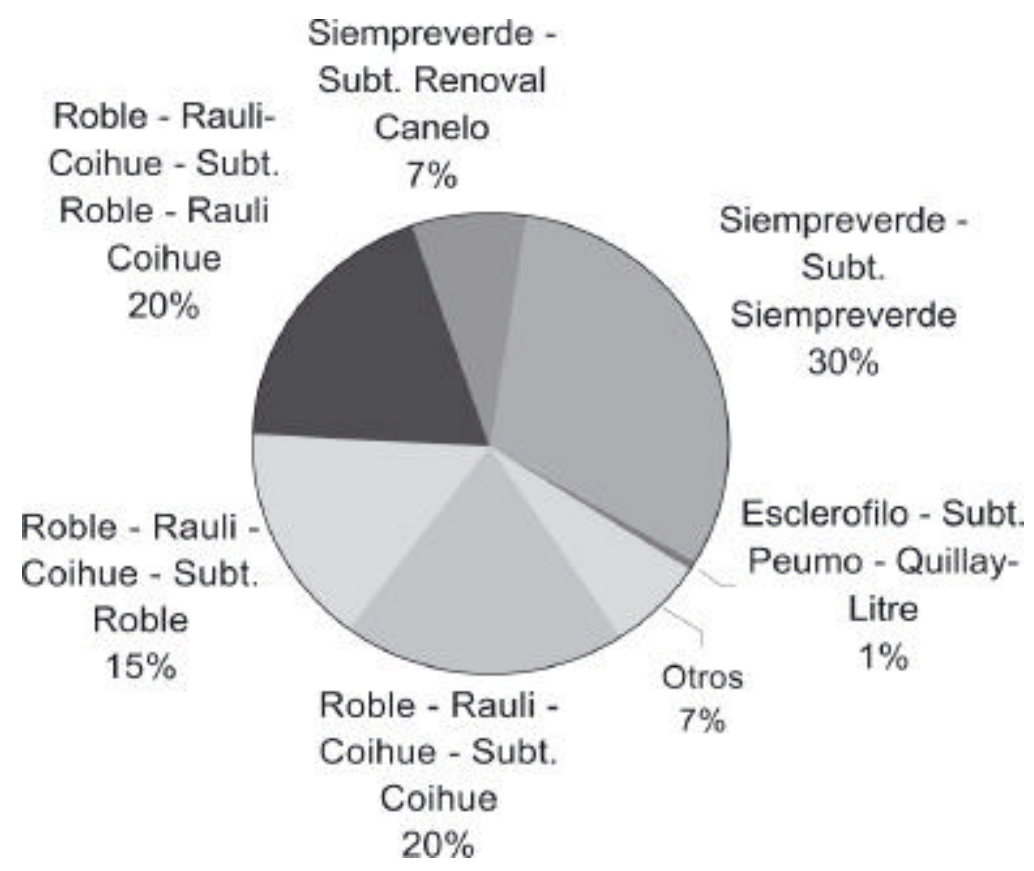

Figura 3. Frecuencia de los registros de B. corallina según tipo y subtipo forestal.

Figure 3. Proportion of $B$. corallina records by forest type and sub-type. 
Al observar las distancias mínimas entre cada uno de los puntos de B. corallina, destaca el hecho que el $71,5 \%$ de los registros presenta puntos próximos a distancias menores a $1.000 \mathrm{~m}$ y el $91,1 \%$ muestran puntos cercanos a una distancia menor a $2.000 \mathrm{~m}$ (Tabla I).

DIVERSIDAD DE ESPECIES ACOMPAÑANTES

La diversidad de especies vegetales registradas coexistentes junto a $B$. corallina es muy variada dependiendo de las comunidades forestales donde se encuentre (Tabla II). Destacan por su dominancia las especies arbóreas Aextoxicon punctatum Ruiz \& Pav. (olivillo), Nothofagus dombeyi (Mirb.) Oerst. (coihue), Nothofagus obliqua (Mirb.) Oerst. (roble), Amomyrtus luma (Molina) D.Legrand \& Kausel (luma), Drimys winteri J.R.Forst. \& G.Forst. (canelo), Laurelia sempervirens (Ruiz \& Pav.) Tul. (laurel), Laureliopsis philippiana (Looser) Schodde (tepa), Luma apiculata (DC.) Burret (arrayán) y Persea lingue Nees (lingue), así como los arbustos, Rhaphithamnus spinosus (Juss.) Moldenke (huayún), Aristotelia chilensis (Molina) Stuntz (maqui) y Chusquea quila (quila), las trepadoras Cissus striata Ruiz \& Pav. (pilpilvoqui), Boquila trifoliolata (DC.) Decne. (pilpilvoqui), Lapageria rosea Ruiz \& Pav. (copihue) y Luzuriaga radicans
Ruiz \& Pav. (quilineja), Lophosoria quadripinnata (J.F.Gmel.) C.Chr. (ampe o palmilla grande) y varias especies del género Blechnum entre muchas otras (Tabla II), representando una amplia muestra de la diversidad florística de la vertiente oceánica de la cordillera de Nahuelbuta.

Cobertura Vegetal y EXTENSIÓN DE LAS Poblaciones DE BERBERIDOPSIS CORALLINA

Los valores más altos de cobertura específica para michay rojo se presentaron en los puntos 80,82 a 85,118 y 119 , con una cobertura entre 50 y $75 \%$ exclusivamente de la especie, seguidos de los puntos 21, 24, 102, 108 y 109 con una cobertura entre 25 y $50 \%$ y de los registros 98, 99 y 106 con una cobertura de la especie entre 10 y $25 \%$. Para el resto de los puntos se registró una cobertura de la especie menor a $10 \%$.

La extensión registrada con presencia de $B$. corallina en cada punto de registro, varía desde 0,01 ha hasta 17,4 ha (Tabla I). Si se observa la frecuencia de los registros según el área observada con presencia de la especie, resalta una frecuencia mayor de puntos con superficie inferior a 0,25 ha. Por otro lado, sólo un número menor de los registros se observó con una extensión superior a 1 ha (Fig. 4).

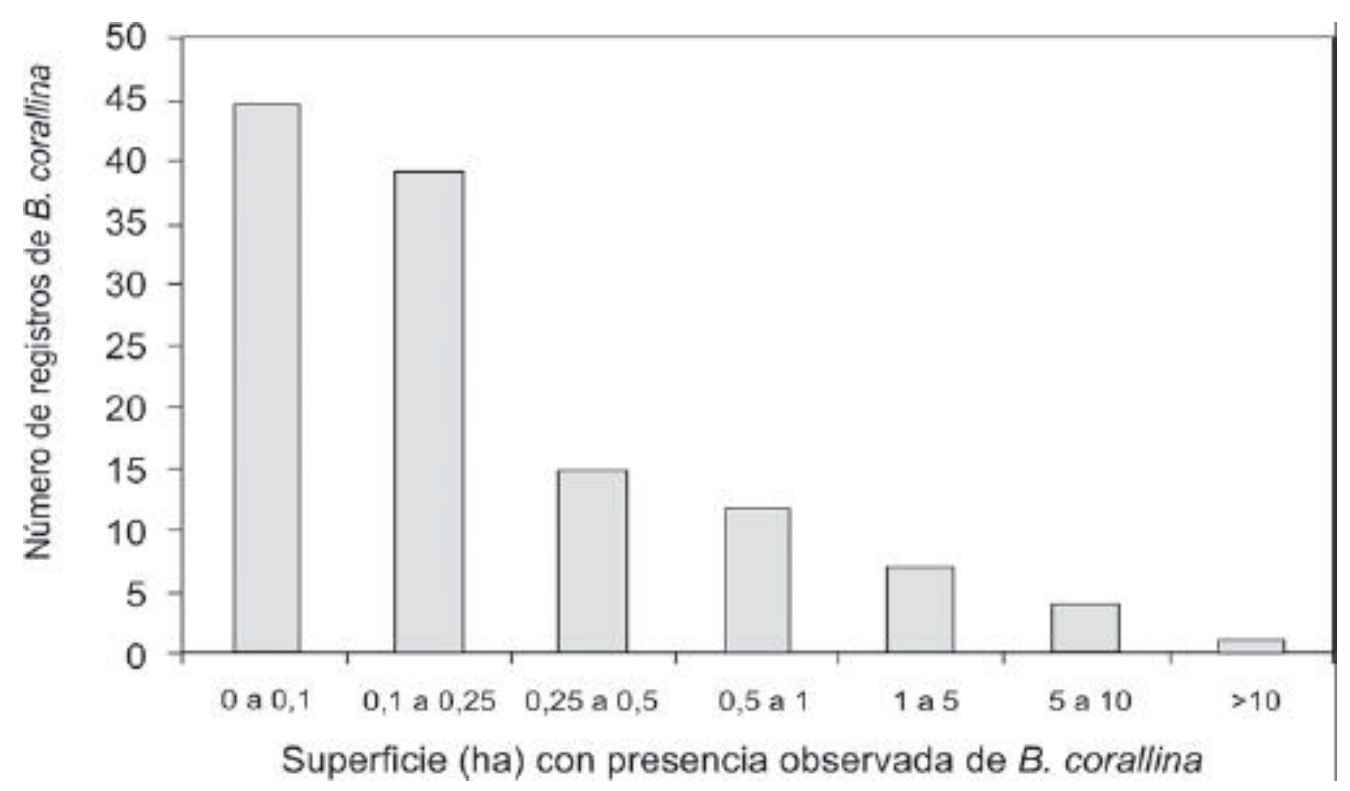

Figura 4. Frecuencia de los registros de B. corallina según superficie observada con presencia de la especie en los fragmentos de vegetación nativa.

FIGURE 4. Number of B. corallina records and patch size of continuous natural vegetation. 
Estos registros se hallan inmersos en fragmentos de bosque nativo generalmente de una mayor extensión, que abarcan desde superficies menores de 1 ha hasta un fragmento de 2653,2 ha (Tabla I). La frecuencia de los registros en rangos de tamaño de los fragmentos respectivos de bosque nativo, muestra una heterogeneidad en su distribución (Fig. 5).

NIVEL DE CONSERVACIÓN DEL HÁBITAT

Los registros de $B$. corallina ubicados en áreas consideradas de alto grado de conservación de hábitat totalizan un $22,8 \%$ de los registros. Aquellos ubicados en situaciones de nivel medio de conservación correspondieron a un $59,3 \%$. Finalmente un $17,9 \%$ de los puntos registrados con la especie se reconocen con un grado bajo comparativo de conservación de hábitat (Fig. 6, Tabla I).

\section{DISCUSION}

Previamente, dentro de la zona de estudio se conocía sólo nueve poblaciones de $B$. corallina, y una de ellas extinta según Le Quesne et al. 2000 y SmithRamírez et al. 2005. Estos nuevos registros presentados aumentan significativamente a aquellos anteriores, dando cuenta de una mayor continuidad geográfica relativa de las poblaciones de $B$. corallina en la zona estudiada. Esto se corrobora al observar las distancias mínimas entre los registros (Tabla I), los que en su gran mayoría presentan puntos cercanos, a distancias menores de $2.000 \mathrm{~m}$, lo que hace pensar en la posibilidad que muchas de ellas se encuentran funcionalmente conectadas en términos de agentes polinizadores como insectos.

Por otro lado, resulta interesante el hallazgo de poblaciones en algunas zonas de las cuales no se tenía referencia cercana, entre las que destacan los registros 69 y 70, ubicados al suroeste de la ciudad de Arauco y el registro 120, localizado al suroeste del lago Lanalhue (Fig. 1, Tabla I). De igual modo, es importante destacar que algunos de estos nuevos registros aumentan el límite altitudinal superior descrito para la especie desde 700 ms.n.m. según Smith-Ramírez et al. 2005, hasta los 885 ms.n.m.

La extensión de los registros observados con B. corallina constituyen superficies pequeñas, en su mayoría menores a 0,25 ha (Tabla I, Fig. 4). Sin embargo, debe notarse que estas poblaciones se hallan contenidas en fragmentos de vegetación nativa de mayor extensión (Tabla I, Fig. 5). Debido a esto, es posible que existan otros puntos de la especie al interior de los mismos fragmentos, en sectores no revisados, lo que implicaría una aún mayor continuidad espacial de esta trepadora endémica al interior de la vegetación nativa estudiada. La heterogeneidad en la distribución del tamaño de los fragmentos de bosque nativo que contienen B.corallina responde en parte al uso histórico del hábitat original de la especie, y resulta notable el hecho que aunque se trate de fragmentos de extensión relativamente pequeña, contribuyen a la conservación de poblaciones de esta especie considerada amenazada.

En este trabajo se utiliza el término poblaciones de la especie, pero se debe tener presente que, tratándose de una enredadera con capacidad de reproducción vegetativa, se desconoce si realmente se trata de poblaciones o grupos de individuos clonales como se ha sugerido por Etisham-Ul-Haq et al. 2001, lo cuál sólo puede ser determinado a través de análisis genéticos. De igual manera, se desconoce si estas poblaciones de michay rojo pudieron haber formado en el pasado un sólo continuo, que se fragmentó con los cambios de uso del suelo.

Ocasionalmente se ha encontrado $B$. corallina creciendo dentro de plantaciones aledañas a fragmentos de bosque nativo con presencia de la especie en la zona de estudio, fenómeno observado también en plantaciones de Pseudotsuga menziesii (Mirb.) Franco en San Juan de la Costa. Se sospecha que estos individuos provienen de crecimiento vegetativo de individuos pre-existentes a la plantación y no de semillas. Ensayos preliminares para evaluar la germinación de semillas de $B$. corallina, tanto dentro del bosque nativo como al interior y borde de plantaciones, no fueron exitosos (SmithRamírez et al. 2005). No obstante, se han observado puntualmente individuos aislados creciendo en taludes y cortes de caminos, no conectados vegetativamente con otros y presumiblemente de origen sexuado. Esto abre la posibilidad de una ocurrencia natural de reproducción por semillas.

Los fragmentos presentados en este estudio constituyen zonas de conservación in situ de la 
biodiversidad presente en los predios de Bosques Arauco S.A. La información de este estudio ha servido de base para generar planes de conservación intensiva en aquellas poblaciones de B. corallina de mayor extensión, al mismo tiempo que la disponibilidad de esta información constituye un importante antecedente para mejorar la planificación de faenas forestales en el área, a fin de fortalecer la protección de los fragmentos de bosque nativo que contienen las poblaciones de esta especie.

La caracterización de la vegetación nativa de los predios de esta empresa, que se distribuyen a lo largo y ancho de la cordillera de Nahuelbuta, reveló numerosos registros nuevos de $B$. corallina. Por esto, es probable la existencia de otros puntos con la especie, en propiedades vecinas que no han sido estudiadas y que presentan similares condiciones de hábitat. Este estudio constituye un ejemplo en que el levantamiento sistemático de información de la vegetación nativa, contribuye a un mejor conocimiento de la presencia y distribución de especies escasas o amenazadas, facilitando la implementación de medidas para su conservación.

Los nuevos registros presentados deberán ser considerados en futuras revisiones de la aplicación del reglamento para la clasificación de especies silvestres (CONAMA 2005) para B corallina, ya que representan un aumento significativo para los factores que determinan su correcta clasificación según la metodología de UICN versión 3.1 (2001), en cuanto a número de registros, extensión de la presencia o área de ocupación de la especie.

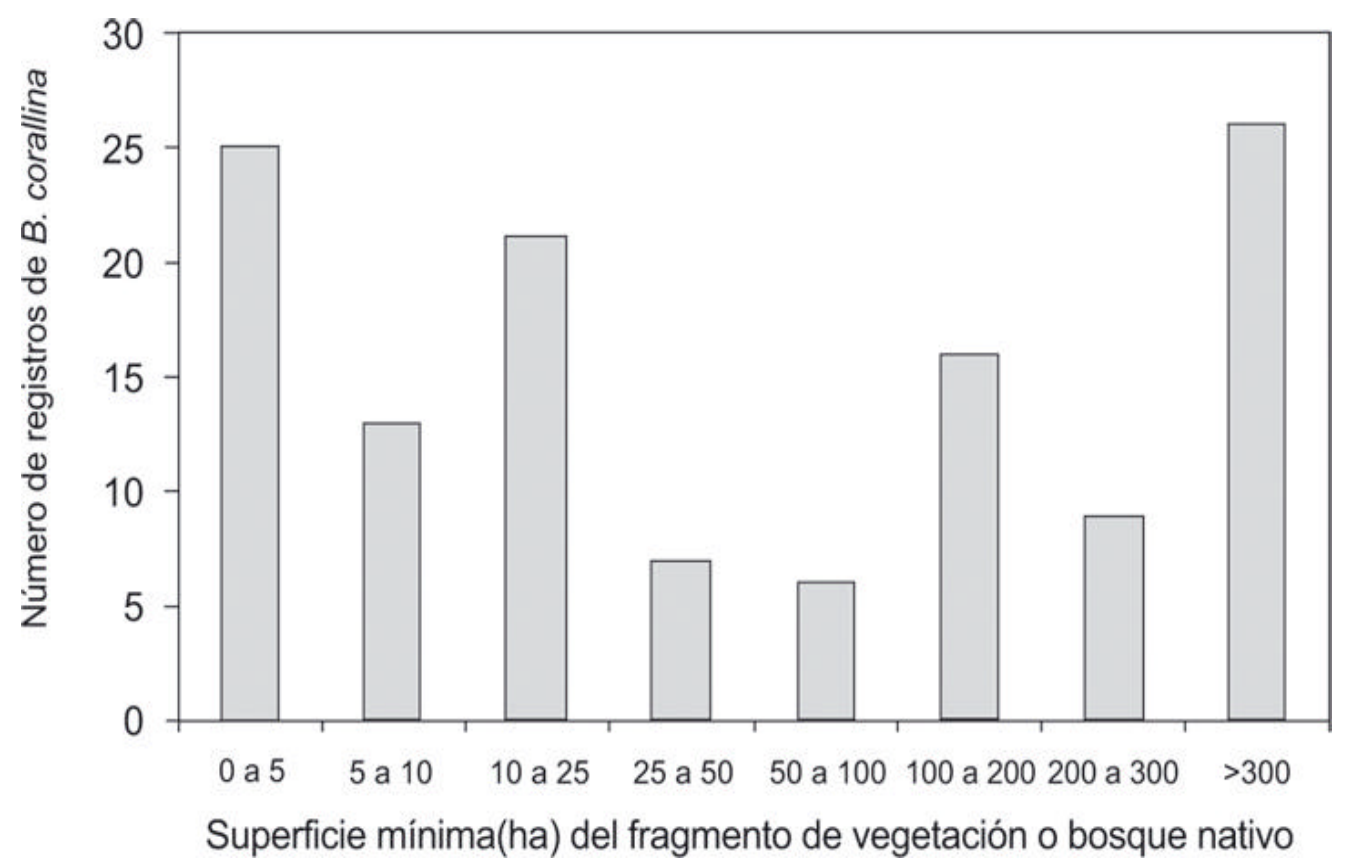

FiguRa 5. Frecuencia de los registros de B. corallina según tamaño de los fragmentos de vegetación nativa donde éstos se encuentran.

FIGURE 5. Number of B. corallina records and patch size of inhabiting plant community. 
Berberidopsis corallina: nuevas poblaciones: AlaRcón, D. ETAL

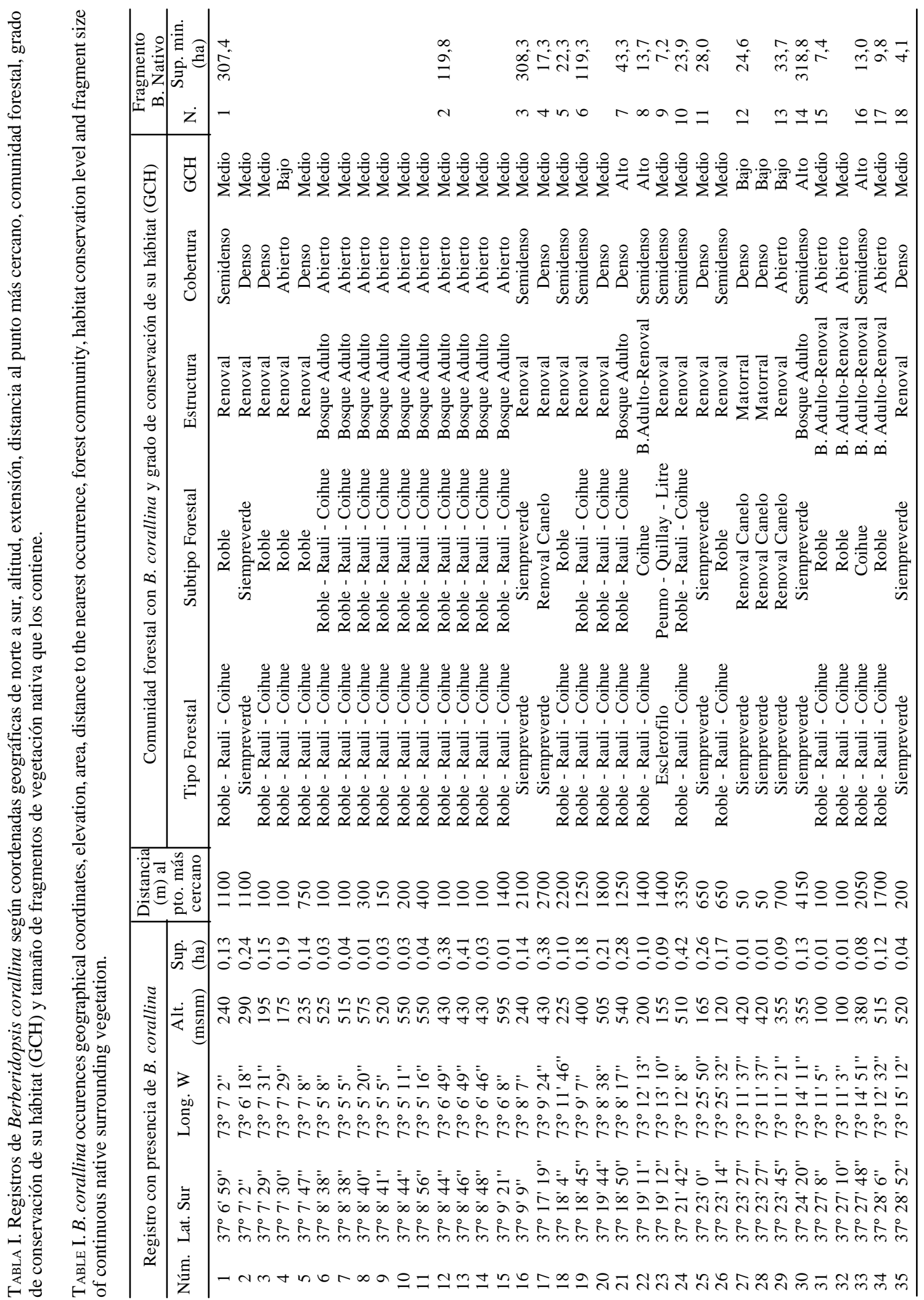




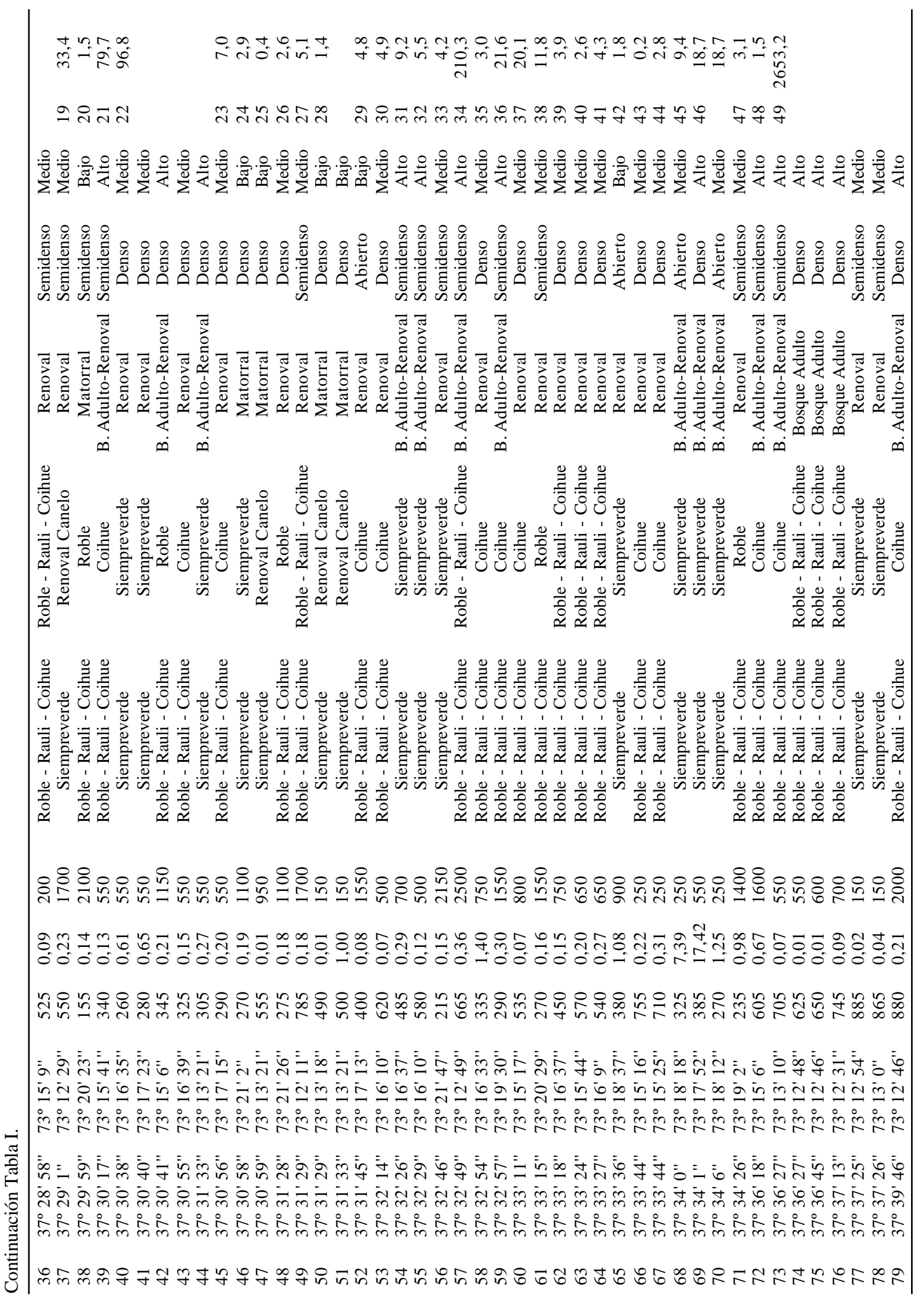


Berberidopsis corallina: nuevas poblaciones: AlaRcón, D. ETAL

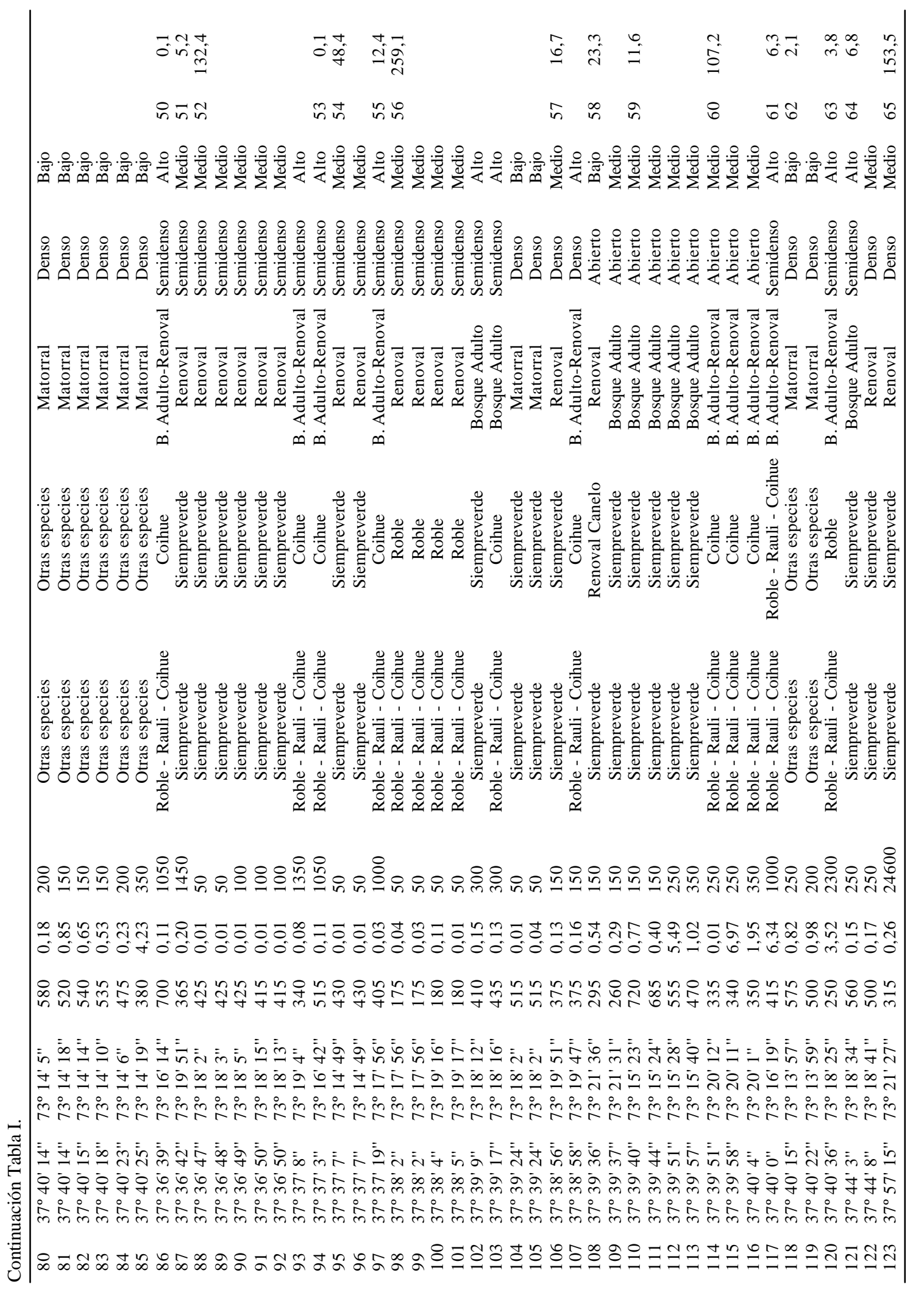


Gayana Bot. 64(2), 2007

TABLA II. Listado de especies de flora asociada a los registros de B. corallina según comunidades forestales, siguiendo clasificación de CONAMA-CONAF-BIRF 1997.

TABLE II. Plant species associated with $B$. corallina records according to forest communities in CONAMA-CONAFBIRF 1997.

\begin{tabular}{|c|c|c|c|c|c|c|c|}
\hline \multirow{2}{*}{ Nombre científico } & \multirow{2}{*}{ Nombre común } & \multicolumn{3}{|c|}{ Tipo Roble-Raulí-Coihue } & \multicolumn{2}{|c|}{ Tipo Siempreverde } & \multirow{2}{*}{ 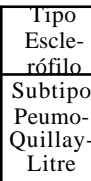 } \\
\hline & & $\begin{array}{l}\text { Subtipo } \\
\text { Roble }\end{array}$ & $\begin{array}{l}\text { Subtipo } \\
\text { Coihue }\end{array}$ & $\begin{array}{c}\text { Subtipo } \\
\text { Roble- } \\
\text { Raulí- } \\
\text { Coihue }\end{array}$ & $\begin{array}{c}\text { Subtipo } \\
\text { Renoval } \\
\text { Canelo }\end{array}$ & $\begin{array}{l}\text { Subtipo } \\
\text { Siempre- } \\
\text { verde }\end{array}$ & \\
\hline Acacia melanoxylon R.Br. & Aromo & & $\mathrm{x}$ & & & $\mathrm{x}$ & \\
\hline Acrisione denticulata (Hook. et Arn.) B.Nord. & Palo de yegua & $\mathrm{x}$ & $\mathrm{x}$ & $\mathrm{x}$ & $\mathrm{x}$ & $\mathrm{x}$ & \\
\hline Adiantum sp. & Palito negro & $\mathrm{x}$ & $\mathrm{x}$ & $\mathrm{x}$ & $\mathrm{x}$ & $\mathrm{x}$ & \\
\hline Aextoxicon punctatum Ruiz et Pav. & Olivillo & $\mathrm{x}$ & $\mathrm{x}$ & $\mathrm{x}$ & $\mathrm{x}$ & $\mathrm{x}$ & $\mathrm{x}$ \\
\hline Amomyrtus luma (Molina) D.Legrand et Kausel & Luma & $\mathrm{x}$ & $\mathrm{x}$ & $\mathrm{x}$ & $\mathrm{x}$ & $\mathrm{x}$ & $\mathrm{x}$ \\
\hline Amomyrtus meli (Phil.) D.Legrand et Kausel & Meli & $\mathrm{x}$ & & & & & \\
\hline Aristotelia chilensis (Molina) Stuntz & Maqui & $\mathrm{x}$ & $\mathrm{x}$ & $\mathrm{x}$ & $\mathrm{x}$ & $\mathrm{x}$ & $\mathrm{x}$ \\
\hline Asplenium dareoides Desv. & Filu-lahuén & $\mathrm{x}$ & $\mathrm{x}$ & $\mathrm{x}$ & & $\mathrm{x}$ & \\
\hline Azara integrifolia Ruiz et Pav. & Challín & $\mathrm{x}$ & $\mathrm{x}$ & & & $\mathrm{x}$ & \\
\hline Azara lanceolata Hook.f. & Aromo & & & $\mathrm{x}$ & & & \\
\hline Baccharis sp. & & & $\mathrm{x}$ & & $\mathrm{x}$ & $\mathrm{x}$ & \\
\hline Berberis sp. & & & & $\mathrm{x}$ & & & \\
\hline Blechnum blechnoides Keyserl. & Iquide & $\mathrm{x}$ & $\mathrm{x}$ & $\mathrm{x}$ & $\mathrm{x}$ & $\mathrm{x}$ & $\mathrm{x}$ \\
\hline Blechnum chilense (Kaulf.) Mett. & Costilla de vaca & $\mathrm{x}$ & $\mathrm{x}$ & $\mathrm{x}$ & $\mathrm{x}$ & $\mathrm{x}$ & $\mathrm{x}$ \\
\hline Blechnum hastatum Kaulf. & Quil-quil & $\mathrm{x}$ & $\mathrm{x}$ & $\mathrm{x}$ & $\mathrm{x}$ & $\mathrm{x}$ & $\mathrm{x}$ \\
\hline Blechnum magellanicum (Desv.) Mett. & Katalapi & $\mathrm{x}$ & $\mathrm{x}$ & $\mathrm{x}$ & $\mathrm{x}$ & $\mathrm{x}$ & $\mathrm{x}$ \\
\hline $\begin{array}{l}\text { Blepharocalyx cruckshanksii (Hook. et Arn.) } \\
\text { Nied. }\end{array}$ & Temu & $\mathrm{x}$ & $\mathrm{x}$ & & $\mathrm{x}$ & & \\
\hline Boquila trifoliolata (DC.) Decne. & Pilpilvoqui & $\mathrm{x}$ & $\mathrm{x}$ & $\mathrm{x}$ & $\mathrm{x}$ & $\mathrm{x}$ & \\
\hline Buddleja globosa Hope & Matico & & $\mathrm{x}$ & $\mathrm{x}$ & & $\mathrm{x}$ & \\
\hline Caldcluvia paniculata (Cav.) D.Don & Tiaca & $\mathrm{x}$ & $\mathrm{x}$ & $\mathrm{x}$ & & $\mathrm{x}$ & \\
\hline Campsidium valdivianum (Phil.) Skottsb. & Pilpilvoqui & $\mathrm{x}$ & $\mathrm{x}$ & $\mathrm{x}$ & & $\mathrm{x}$ & $\mathrm{x}$ \\
\hline Cestrum parqui L'Hérit. & Palqui & $\mathrm{x}$ & & & & & \\
\hline Chusquea culeou Desv. & Colihue & $\mathrm{x}$ & & & & & \\
\hline Chusquea quila Kunth & Quila & $\mathrm{x}$ & $\mathrm{x}$ & $\mathrm{x}$ & $\mathrm{x}$ & $\mathrm{x}$ & $\mathrm{x}$ \\
\hline Cissus striata Ruiz \& Pav. & Pilpilvoqui & $\mathrm{x}$ & $\mathrm{x}$ & $\mathrm{x}$ & $\mathrm{x}$ & $\mathrm{x}$ & \\
\hline Citronella mucronata (Ruiz et Pav.) D.Don & Huillipatagua & & & $\mathrm{x}$ & & & \\
\hline Corynabutilon vitifolium (Cav.) Kearney & Huella & & & & & $\mathrm{x}$ & \\
\hline Cryptocarya alba (Molina) Looser & Peumo & & $\mathrm{x}$ & $\mathrm{x}$ & & & $\mathrm{x}$ \\
\hline Dasyphyllum diacanthoides (Less.) Cabrera & Trevo & $\mathrm{x}$ & $\mathrm{x}$ & $\mathrm{x}$ & & $\mathrm{x}$ & $\mathrm{x}$ \\
\hline Desfontainia spinosa Ruiz et Pav. & Taique & $\mathrm{x}$ & & $\mathrm{x}$ & $\mathrm{x}$ & $\mathrm{x}$ & \\
\hline Drimys winteri J.R.Forst. et G.Forst. & Canelo & $\mathrm{x}$ & $\mathrm{x}$ & $\mathrm{x}$ & $\mathrm{x}$ & $\mathrm{x}$ & $\mathrm{x}$ \\
\hline Elytropus chilensis (A.DC.) Müll.Arg. & Quilmay & $\mathrm{x}$ & $\mathrm{x}$ & $\mathrm{x}$ & & $\mathrm{x}$ & \\
\hline Embothrium coccineum J.R.Forst. et G.Forst. & Notro & & $\mathrm{x}$ & $\mathrm{x}$ & $\mathrm{x}$ & $\mathrm{x}$ & \\
\hline Escallonia alpina Poepp. et Endl. & Nipa & & $\mathrm{x}$ & $\mathrm{x}$ & & & \\
\hline Eucalyptus globulus Labill. & Eucalipto & & $\mathrm{x}$ & & & & \\
\hline Eucryphia cordifolia Cav. & Ulmo & $\mathrm{x}$ & $\mathrm{x}$ & $\mathrm{x}$ & $\mathrm{x}$ & $\mathrm{x}$ & $\mathrm{x}$ \\
\hline Fuchsia magellanica Lam. & Chilco & $\mathrm{x}$ & $\mathrm{x}$ & $\mathrm{x}$ & & $\mathrm{x}$ & \\
\hline Galium hypocarpium (L.) Endl. ex Griseb. & Relbún & $\mathrm{x}$ & & & & & \\
\hline Gaultheria insana (Molina) D.J.Middleton & Hued-hued & & & $\mathrm{x}$ & & & \\
\hline Gaultheria phillyreifolia (Pers.) Sleumer & Chaura & $\mathrm{x}$ & $\mathrm{x}$ & $\mathrm{x}$ & $\mathrm{x}$ & $\mathrm{x}$ & \\
\hline Gevuina avellana Molina & Avellano & $\mathrm{x}$ & $\mathrm{x}$ & $\mathrm{x}$ & $\mathrm{x}$ & $\mathrm{x}$ & $\mathrm{x}$ \\
\hline Gomortega keule (Molina) Baill. & Queule & & $\mathrm{x}$ & & & & \\
\hline
\end{tabular}


Berberidopsis corallina: nuevas poblaciones: Alarcón, D. etaL

Continuación Tabla II.

\begin{tabular}{|c|c|c|c|c|c|c|c|}
\hline Greigia sphacelata (Ruiz et Pav.) Regel & Chupón & $\mathrm{x}$ & $\mathrm{x}$ & $\mathrm{x}$ & $\mathrm{x}$ & $\mathrm{x}$ & \\
\hline Hydrangea serratifolia (Hook. et Arn.) F.Phil. & Canelilla & & & $\mathrm{x}$ & & $\mathrm{x}$ & \\
\hline Hymenophyllum sp. & Helecho película & & $\mathrm{x}$ & $\mathrm{x}$ & & & \\
\hline Hypolepis poeppigii (Kunze) R.A.Rodr. & Wilel-lahuén & & & $\mathrm{x}$ & & & \\
\hline Lapageria rosea Ruiz et Pav. & Copihue & $\mathrm{x}$ & $\mathrm{x}$ & $\mathrm{x}$ & $\mathrm{x}$ & $\mathrm{x}$ & $\mathrm{x}$ \\
\hline Lardizabala biternata Ruiz et Pav. & Coguil & $\mathrm{x}$ & $\mathrm{x}$ & $\mathrm{x}$ & & $\mathrm{x}$ & \\
\hline Laurelia sempervirens (Ruiz et Pav.) Tul. & Laurel & $\mathrm{x}$ & $\mathrm{x}$ & $\mathrm{x}$ & $\mathrm{x}$ & $\mathrm{x}$ & $\mathrm{x}$ \\
\hline Laureliopsis philippiana (Looser) Schodde & Tepa & $\mathrm{x}$ & $\mathrm{x}$ & $\mathrm{x}$ & $\mathrm{x}$ & $\mathrm{x}$ & $\mathrm{x}$ \\
\hline Loasa sp. & & & & & & $\mathrm{x}$ & \\
\hline Lobelia tupa $\mathrm{L}$. & Tupa, Tabaco del diablc & & & & $\mathrm{x}$ & & \\
\hline Lomatia dentata (Ruiz et Pav.) R.Br. & Piñol, Avellanillo & $\mathrm{x}$ & $\mathrm{x}$ & $\mathrm{x}$ & $\mathrm{x}$ & $\mathrm{x}$ & $\mathrm{x}$ \\
\hline Lomatia ferruginea (Cav.) R.Br. & Fuinque & $\mathrm{x}$ & & $\mathrm{x}$ & $\mathrm{x}$ & $\mathrm{x}$ & \\
\hline Lomatia hirsuta (Lam.) Diels ex J.F.Macbr. & Radal & $\mathrm{x}$ & $\mathrm{x}$ & $\mathrm{x}$ & & $\mathrm{x}$ & \\
\hline Lophosoria quadripinnata (J.F.Gmel.) C.Chr. & Ampe & $\mathrm{x}$ & $\mathrm{x}$ & $\mathrm{x}$ & $\mathrm{x}$ & $\mathrm{x}$ & \\
\hline Luma apiculata (DC.) Burret & Arrayán & $\mathrm{x}$ & $\mathrm{x}$ & $\mathrm{x}$ & $\mathrm{x}$ & $\mathrm{x}$ & $\mathrm{x}$ \\
\hline Luma chequen (Molina) A.Gray & Chequén & $\mathrm{x}$ & & & $\mathrm{x}$ & & \\
\hline Luzuriaga radicans Ruiz et Pav. & Quilineja & $\mathrm{x}$ & $\mathrm{x}$ & $\mathrm{x}$ & $\mathrm{x}$ & $\mathrm{x}$ & $\mathrm{x}$ \\
\hline Maytenus magellanica (Lam.) Hook.f. & Leña dura & $\mathrm{x}$ & & & & & \\
\hline $\begin{array}{l}\text { Megalastrum spectabile (Kaulf.) A.R.Sm. et } \\
\text { R.C.Moran }\end{array}$ & Pesebre & $\mathrm{x}$ & $\mathrm{x}$ & & $\mathrm{x}$ & $\mathrm{x}$ & \\
\hline Mitraria coccinea $\mathrm{Cav}$. & Botellita & & & $\mathrm{x}$ & & $\mathrm{x}$ & $\mathrm{x}$ \\
\hline Mutisia sp. & Clavel del campo & & & $\mathrm{x}$ & $\mathrm{x}$ & & \\
\hline Myoschilos oblonga Ruiz et Pav. & Orocoipo & & $\mathrm{x}$ & & $\mathrm{x}$ & $\mathrm{x}$ & \\
\hline Myrceugenia exsucca (DC.) O.Berg & Pitra & $\mathrm{x}$ & $\mathrm{x}$ & & $\mathrm{x}$ & $\mathrm{x}$ & \\
\hline Myrceugenia leptospermoides (DC.) Kausel & Macolla & & & & & $\mathrm{x}$ & \\
\hline Myrceugenia planipes (Hook. et Arn.) O.Berg & Picha-picha & $\mathrm{x}$ & $\mathrm{x}$ & & & $\mathrm{x}$ & \\
\hline Nertera granadensis Druce & Rucachucao & $\mathrm{x}$ & & $\mathrm{x}$ & $\mathrm{x}$ & & \\
\hline Nothofagus dombeyi (Mirb.) Oerst. & Coihue & $\mathrm{x}$ & $\mathrm{x}$ & $\mathrm{x}$ & $\mathrm{x}$ & $\mathrm{x}$ & \\
\hline Nothofagus nervosa Phil. & Raulí & & $\mathrm{x}$ & $\mathrm{x}$ & $\mathrm{x}$ & $\mathrm{x}$ & \\
\hline Nothofagus obliqua (Mirb.) Oerst. & Roble & $\mathrm{x}$ & & $\mathrm{x}$ & $\mathrm{x}$ & $\mathrm{x}$ & $\mathrm{x}$ \\
\hline Persea lingue Nees & Lingue & $\mathrm{x}$ & $\mathrm{x}$ & $\mathrm{x}$ & $\mathrm{x}$ & $\mathrm{x}$ & $\mathrm{x}$ \\
\hline Peumus boldus Molina & Boldo & & $\mathrm{x}$ & $\mathrm{x}$ & & $\mathrm{x}$ & \\
\hline Pinus radiata D.Don & Pino insigne & & & & $\mathrm{x}$ & $\mathrm{x}$ & \\
\hline Podanthus mitiqui Lindl. & Mitique & $\mathrm{x}$ & & & & & \\
\hline Podocarpus salignus D.Don & Mañío de hojas largas & $\mathrm{x}$ & $\mathrm{x}$ & $\mathrm{x}$ & & $\mathrm{x}$ & $\mathrm{x}$ \\
\hline Pseudopanax laetevirens (Gay) Franch. & Sauco del diablo & $\mathrm{x}$ & & $\mathrm{x}$ & $\mathrm{x}$ & $\mathrm{x}$ & \\
\hline Retanilla ephedra (Vent.) Brongn. & Retamilla & & & & & $\mathrm{x}$ & \\
\hline Rhamnus diffusus Clos & Palo negro & & & $\mathrm{x}$ & & $\mathrm{x}$ & \\
\hline Rhaphithamnus spinosus (Juss.) Moldenke & Huayún & $\mathrm{x}$ & $\mathrm{x}$ & $\mathrm{x}$ & $\mathrm{x}$ & $\mathrm{x}$ & $\mathrm{x}$ \\
\hline Ribes valdivianum Phil. & Parrilla & & & & & $\mathrm{x}$ & \\
\hline Rubus ulmifolius Schott & Zarzamora & $\mathrm{x}$ & $\mathrm{x}$ & & & & $\mathrm{x}$ \\
\hline Sarmienta scandens Pers. & Medallita & $\mathrm{x}$ & $\mathrm{x}$ & $\mathrm{x}$ & & $\mathrm{x}$ & \\
\hline Saxegothaea conspicua Lindl. & Mañío hembra & $\mathrm{x}$ & & $\mathrm{x}$ & & & \\
\hline Teline monspessulana (L.) K.Koch & Retamo & $\mathrm{x}$ & $\mathrm{x}$ & $\mathrm{x}$ & $\mathrm{x}$ & $\mathrm{x}$ & \\
\hline Ugni molinae Turcz. & Murtilla & $\mathrm{x}$ & $\mathrm{x}$ & $\mathrm{x}$ & $\mathrm{x}$ & $\mathrm{x}$ & \\
\hline Ulex europaeus L. & Espino & & & & $\mathrm{x}$ & & \\
\hline Uncinia sp. & & $\mathrm{x}$ & $\mathrm{x}$ & $\mathrm{x}$ & $\mathrm{x}$ & $\mathrm{x}$ & \\
\hline Viola sp. & & & $\mathrm{x}$ & & & & \\
\hline Weinmannia trichosperma Cav. & Tineo & $\mathrm{x}$ & $\mathrm{x}$ & $\mathrm{x}$ & & $\mathrm{x}$ & \\
\hline
\end{tabular}




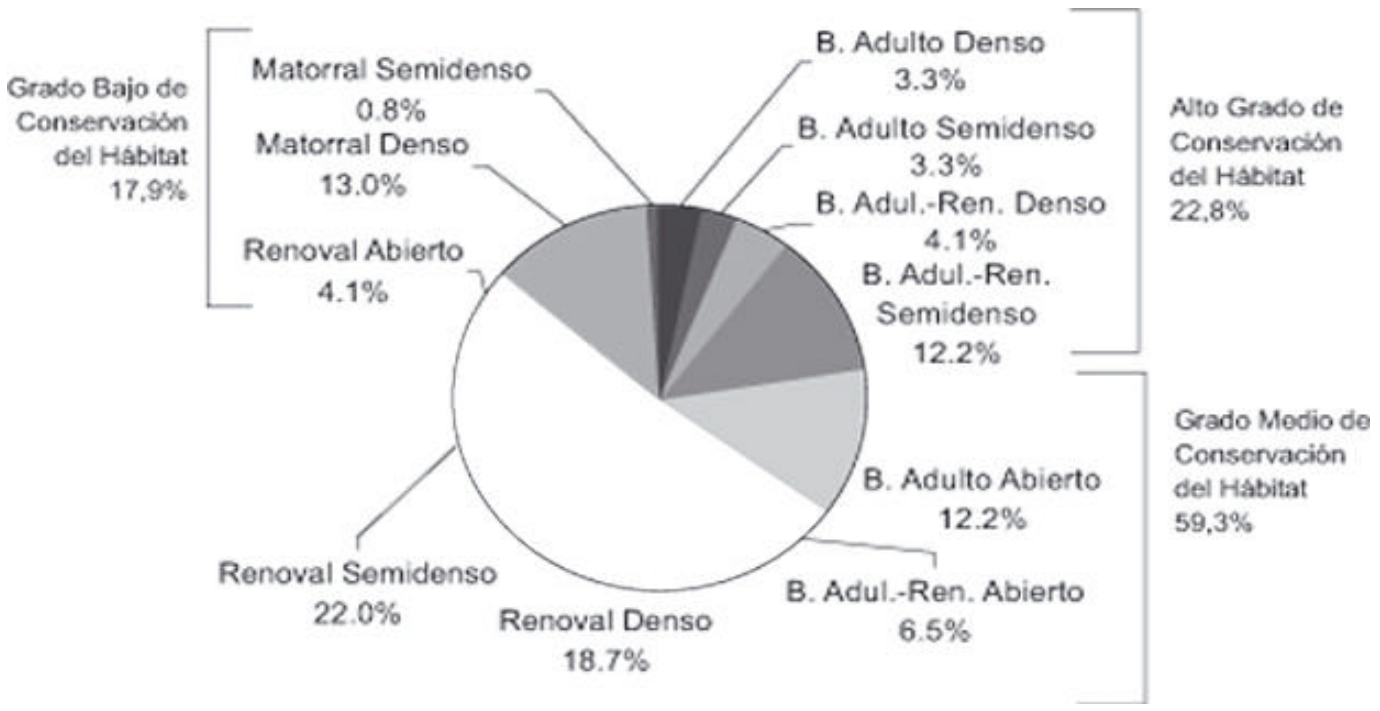

Figura 6. Frecuencia de los registros de $B$. corallina según estructura, cobertura y grado de conservación del hábitat.

FIGURE 6. Proportion of B. corallina records by forest structure, canopy cover and conservation status of its habitat.

\section{AGRADECIMIENTOS}

D. Alarcón y M. Oliva agradecen el apoyo de Bosques Arauco S.A. y Bioforest S.A. y comentarios de Eduardo Rodríguez. C. Smith-Ramírez agradece a Fundación BBVA, Fondecyt-Fondap 1501-0001 (Programa 3), Centro CASEB de P. Universidad Católica de Chile y al Proyecto Núcleo Milenio 99103 FICM.

\section{BIBLIOGRAFIA}

APG II - The Angiosperm Phylogeny Group. 2003. An update of the angiosperm phylogeny group classification for the orders and families of flowering plants: APG II. Botanical Journal of the Linnean Society 141: 399-436.

Benoit, I. (Ed.) 1989. Libro rojo de la flora terrestre de Chile. Corporación Nacional Forestal, Santiago. $157 \mathrm{pp}$.

CONAF-CONAMA-BIRF. 1997. Catastro y evaluación de los recursos vegetacionales nativos de Chile. Informe nacional con variables ambientales, Santiago. 90 p.
CONAMA 2005. Proceso de clasificación de especies silvestres 2005. Comisión Nacional del Medio Ambiente. URL: http://www.conama.cl/ clasificacionespecies/index.htm. Acceso: Abril 28, 2006.

Etisham-Ul-Haq, M., T.R. Allnutt, C. Smith-Ramírez, M.F. Gardner, J.J. Armesto \& A.C. Newton. 2001. Patterns of Genetic Variation in in and ex situ Populations of the threatened Chilean Vine Berberidopsis corallina, Detected Using RAPD Markers. Annals of Botany 87: 813-821.

Hajek, E.R. \& F. Di Castri. 1975. Bioclimatografía de Chile. Universidad Católica de Chile, Santiago. Bioclimatografía de Chile. Dirección de Investigación. Universidad Católica de Chile. $225 \mathrm{pp}$

Hechenleitner, P., M.F. Gardner, P.I. Thomas, C. Echeverría, B. Escobar, P. Brownless \& C. M ARTínez. 2005. Plantas Amenazadas del CentroSur de Chile. Distribución, Conservación y Propagación. Primera Edición. Universidad Austral de Chile y Real Jardín Botánico de Edimburgo. 188 pp.

Le Quesne, C., R. Carrasco \& L. Sandoval. 2000. Lista de puntos florísticos de algunas especies en peligro, raras y vulnerables en la Región del BíoBío. CONAF Serie Técnica Año 3 (4): 1-16. 
Berberidopsis corallina: nuevas poblaciones: Alarcón, D. etaL

Riedemann, P. \& G. Aldunate. 2004. Flora nativa de valor ornamental. Identificación y Propagación. Chile Zona Sur. Editorial Andrés Bello. 516 pp.

Ronse De Craene, L. 2004. Floral development of Berberidopsis corallina: a crucial link in the evolution of flowers in the core eudicots. Annals of Botany 94: 741-751.

Serra, M.T., R. Gajardo \& A. Cabello. 1986. Berberidopsis corallina Hook.f. Programa de protección y recuperación de la flora nativa de Chile. Ficha técnica de especies amenazadas. Corporación Nacional Forestal. 17 pp.

Smith-Ramírez, C. 1995. Algunos usos indígenas tradicionales de la flora del bosque templado. En: Ecología de los bosques nativos de Chile (Eds. J.J. Armesto, C. Villagrán \& M.K. Arroyo), pp. 389-404. Editorial Universitaria, Santiago.
Smith-Ramírez, C., B. Campillo, J.L. Célis-Diez \& M.F. GARDNER. 2005. Historia natural de la enredadera endémica Berberidopsis corallina. En: Historia, biodiversidad y ecología de los bosques costeros de Chile (Eds. C. Smith-Ramírez, J.J. Armesto \& C. Valdovinos), pp. 284-288. Editorial Universitaria, Santiago.

UICN. 2001. Categorías y Criterios de la Lista Roja de la UICN: Versión 3.1. Comisión de Supervivencia de Especies de la UICN. Unión Internacional para la Conservación de la Naturaleza y Recursos Naturales, Gland, Suiza y Cambridge, Reino Unido. $33 \mathrm{pp}$.

Walter, K. S. \& H. Gillett. (Eds.) 1998. 1997 IUCN Red List of Threatened Plants. Compiled by the World Conservation Monitoring Centre. IUCN, Gland, Switzerland and Cambridge, UK. 862 pp. 